\title{
The role of mitochondria in cellular toxicity as a potential drug target
}

\author{
Duojiao Wu $\cdot$ Xiangdong Wang $\cdot$ Hongzhi Sun
}

Received: 31 December 2017 / Accepted: 13 February 2018 / Published online: 6 March 2018

(C) Springer Science+Business Media B.V., part of Springer Nature 2018

\begin{abstract}
Mitochondrial malfunction is related to aging and to the onset of many diseases, such as obesity/ diabetes, cancer, and cardiovascular and neurodegenerative diseases. The molecular principles of biological and toxicological processes the mitochondria can regulate should be disease-specific, cell type-specific, and drug targetable. Mitochondrial biology and toxicology is evolving and undergoing a revolution through fastdeveloping biotechnologies garnering increasing attention due to the importance of targeted therapies. Mitochondrial energy production and metabolism are conducted via post-mitochondrial signaling, and are controlled by extra-mitochondrial pathways. Mitochondrial biology and toxicology has a history spanning over 30 years and is one of the main scientific focuses at Cell Biology and Toxicology. It is our aim to pioneer innovations of mitochondrial biology and toxicology to improve the understanding, highlight the latest development, and find mitochondria-based targets for therapies. It is expected to know how drugs can initiate
\end{abstract}

D. Wu $(\bowtie) \cdot X$. Wang

Zhongshan Hospital Institute of Clinical Bioinformatics, Shanghai Institute of Clinical Bioinformatics, University Shanghai Medical School, Shanghai, China

e-mail:wuduojiao@126.com

X. Wang

e-mail: xiangdong.wang@ clintransmed.org

H. Sun

Jinzhou Medical University, Jinzhou, Liaoning Province, China e-mail: cmushz@163.com mitochondrial dysfunction, the role of nuclear messages in regulating mitochondrial DNA (MtDNA), and how mitochondria communicate between or with other cells. Further studies are crucial to discover how mitochondria control the process of immune response, autophagy/ mitophagy, genome activation, and cell interaction. It is also needed to innovate how the transcription is started and terminated within mitochondria, the cytosolic proteins and other organelles interact with mitochondria, and MtDNA regulates the function of mitochondrial respiratory megacomplexes.

Keywords Mitochondria $\cdot$ MtDNA $\cdot$ Metabolism . Signals $\cdot$ Regulation

Mitochondria are organelles of a eukaryote cell. They make most of the supply of adenosine triphosphate (ATP). Mitochondria are known as "the powerhouse of the cell." In addition to supplying cellular energy, mitochondria are involved in a range of other processes, such as signaling, cellular differentiation, cell death, and the control of the cell division cycle and cell growth. Mitochondrion is immediately thought of when discussing mitochondrial dysfunction in drug toxicology. An unexpected number of molecular communications and crosstalk are required to achieve each interaction and function in the mitochondria. We should consider the molecular principles of biological and toxicological processes the mitochondria controls and participates in; then, to measure mitochondrial functional phenotypes, e.g. production of reactive oxygen species and 
metabolites, enzyme activities, and altered membrane potentials. We should consider whether those principles are disease-specific, function-specific, cell type-specific, and drug targetable. Mitochondrial biology and toxicology is evolving and undergoing a revolution through fast-developing biotechnologies garnering increasing attention due to the importance of targeted therapies. Mitochondrial biology and toxicology has a history spanning over 30 years and is one of the main scientific focuses at Cell Biology and Toxicology. It is our aim to pioneer innovations of mitochondrial biology and toxicology, in order to improve the understanding, highlight the latest development, and find mitochondria-based targets for therapies.

\section{Thinking of mitochondrial DNA}

Most of DNA is in the cell nucleus, while the mitochondrion has its own independent genome. Further, its DNA shows substantial similarity to bacterial genomes. Chemicals can induce ganglion cell axonal degeneration by altering the motility of axonal mitochondria (Kikuchi et al. 2017). Antiretroviral therapy has dramatically reduced mortality in human immunodeficiency virus (HIV) infection. However, the antiretroviral drug is the potential cause of muscle pathology in HIV-infected persons demonstrating acquired mitochondrial dysfunction (Gardner et al. 2014). Mitochondrial homeostasis influences the tension of cancer cell stress responses regulated by the Hippo-macrophage stimulating 1 pathway-activated c-Jun N-terminal kinase/p53 pathway, leading to BCL2 interacting protein 3-related mitophagy (Li et al. 2017). Efavirenz, a nonnucleoside reverse transcriptase inhibitor, could alter mitochondrial function, transmembrane potential, and morphology, and lead to cell toxicity through depolarization of mitochondrial transmembrane potential and a cascade of events causing cell death, after the drug entered the mitochondrial compartment (Ganta et al. 2017). Cell stress responses via mitochondria occur in both physiological and pathophysiological conditions, during drug actions, or in the process of Cell Biology and Toxicology (CBT). Mitochondria can be an important control driver in the production of cell energy regulated by the MtDNA. MtDNA acts as the command control center to schedule energetic productions and maintain cell metabolisms and molecular diversification within the mitochondria, responsible for the development of diseases (Sun et al. 2017; Lv et al. 2017). It is clear that the mitochondria function as the energy motor and control a series of organelle responses and signal pathways in CBT, while a large number of intracellular or extracellular elements can influence, regulate, or control the mitochondrial function. More efforts and investigations are urgently required to explore molecular mechanisms to clarify whether the mitochondria control downstream energy and metabolism or if it is controlled by upstream signals. MtDNA cannot be ignored when we discuss about mitochondrial function and cell metabolism as response to drugs and frequently plays a pivotal role.

Importantly, although the genomes are physically distinct, mitochondrial function must rely on an orchestrated cross talk between nuclear and mitochondrial genes. The nucleus-encoded proteins instruct the import of mitochondrial proteins and the correct assembly of OXPHOS complexes. Nonetheless, the regulation of mitochondrial activity requires a bidirectional flow of information. The nuclear gene expression is influenced by signals coming from mitochondria through retrograde communication. Therefore, we should consider the role of the cross talk between the nucleus-mitochondrion communication in cellular toxicity.

\section{Bridging the crosstalk}

MtDNA is a central operator that regulates and bridges the down- or up-stream signals of mitochondrial function, during which MtDNA can act as the receptor to receive major signals from extra-mitochondria (physiological or environmental alterations, etc.) and minor signals from the feedback of intra-mitochondrial metabolism (ATP production, etc.). For example, ubiquitinspecific peptidase 30 (USP30) is a mitochondrial deubiquitinase which controls the quality and homeostasis of mitochondrial function. One of its most essential functions is that USP30 over-activation can initiate the mitochondrial dynamic signaling and result in mutations or sequence aberrations of MtDNA through the mediated deubiquitination of ubiquitylated forms of mitofusins and the removal of Ub chains from Lys 6 and Lys 11 on mitochondria-derived proteins (Hou et al. 2017). Mitochondria can be directly or indirectly targeted as receptors in pharmacokinetics, dynamics, metabolism, and efficacy of drugs. Of those, Quisinostat 
as a novel second-generation histone deacetylase inhibitor inhibited cancer cell growth and epithelialmesenchymal transition by altering mitochondrial function (Bao et al. 2016). This particular study failed to provide evidence on whether the upregulated acetylation of histones $\mathrm{H} 3$ and $\mathrm{H} 4$, non-histone protein $\alpha$ tubulin, p53 acetylation at K382/K373 sites, or the expression of $\mathrm{p} 21$ (Waf1/Cip1) are the initiator of Quisinostat-induced mitochondrial dysfunction per se or if it is the key player of mitochondria-driven cancer cell cease. It is no doubt that the MtDNA plays the critical role in the cross talk between the cellular membrane and mitochondria, between mitochondria and nucleus, or between mitochondrial products and autophagy/mitophagy (Medvedev et al. 2017).

\section{Controlling mitochondrial characteristics}

MtDNA plays the decisive role in the regulation and control of mitochondria-driven cell biology and toxicology as well as diseases. With the improvement of methodologies and bioethics of MtDNA sequencing, it is possible to understand roles of MtDNA heterogeneity and mutation in the regulation of mitochondrial function and in pathogenesis of diseases, and apply MtDNA sequencing for monitoring mitochondrial dysfunction and therapeutic effects (Qian et al. 2017). Alterations of MtDNA copy number and mutations were proposed as potential biomarkers to monitor severity, duration, stage, response to therapy, and prognosis in patients with lung cancer (Liu et al. 2017). It remains unclear how MtDNA variations are involved in carcinogenesis and development of lung cancer and contribute lung cancer therapies. For example, cellular survival signal transduction systems protect mitochondrial integrity against druginduced stress; Meanwhile, apoptosis-related molecules are orchestrated around mitochondria to induce and execute apoptosis in cancer cells. These stress pathways and molecules play important roles in the development of drug resistance in cancer cells. It is therefore intriguing to explore how the cross talk of apoptosis-related molecules regulates various mitochondrial functions leading to drug resistance. Figure 1 illustrated the drug-induced stress pathways and drug toxicities within the mitochondrion. MtDNA mutations, e.g., insertion, deletion, or point mutations, occur in response to carcinogenetic factors, drugs, and stress; influence the immune responses by the changes of immune cell metabolism; alter cell differentiation and evolution during tissue repair and MtDNA stability during DNA repair; and hypersensitize cells to challenges (Wang et al. 2017a, b; Song et al. 2017; Gao et al. 2017; Zhang et al. 2017).

\section{Initiating and terminating the signaling}

MtDNA can be transferred between cells as a signaling message to provoke the activation of cell or act as an antigen to be copied and amplified for cell sensitivities. $\mathrm{Xu}$ et al. (2017) recently found that MtDNA released from tumor cells was harvested by dendritic cells with the assistance of CD47-signal regulatory protein $\alpha$ interaction and recognized by cyclic-GMP-AMP synthase in the dendritic cell cytosol. Tumor nuclear DNA in the circulation or leukocytes were suggested to be clinical biomarkers to all driver DNA alterations present in matched metastatic tissue, since there is evidence that most of the circulating tumor DNA were consistent with the changes in tumor tissue DNA (Wyatt et al. 2017). Cytosolic tumor MtDNA in dendritic cells acts as antigens to overproduce type I interferon and form antitumor-adaptive immunity (Xu et al. 2017). Such a process is highly dependent upon CD47 activation, since the inhibition/insufficiency of CD47 results in the over-activation of NADPH oxidase NOX2, low phagosomal acidification, or reduced degradation of MtDNA. In human mitochondria, the transcription termination occurs at a G-quadruplex region near the replication origin after the RNA polymerase mitochondrial bonds with the mitochondrial transcription elongation factor by interactions of the dimeric pseudonuclease core of the factor with mobile structural elements in the polymerase (Hillen et al. 2017). This specific factor can bind with DNA and the RNA exit channel to terminate synthesis of the replication primer on basis of the formation of the RNA G-quadruplex structure. It should be further explored whether the variation between nuclear and mitochondrial DNA and degrees of MtDNA mutations may influence the specificity and sensitivity of MtDNA or the processes of MtDNA replication, transcription, and termination. In addition to the measurement of mitochondrial function after drug delivery, drugs can induce micronuclei formation, chromosomal aberrations, cytostasis, and mitochondrial-mediated apoptosis as part of the cytogenetic toxicity in CBT (Singireesu et al. 2017). 
Fig. 1 Drug-induced stress pathways and drug toxicities within the mitochondrion. Cellular survival signal transduction systems protect mitochondrial integrity against drug-induced stress. Meanwhile, apoptosis-related molecules are orchestrated around mitochondria to induce and execute apoptosis in cancer cells. These pathways and molecules play important roles in the development of drug resistance in cancer cells

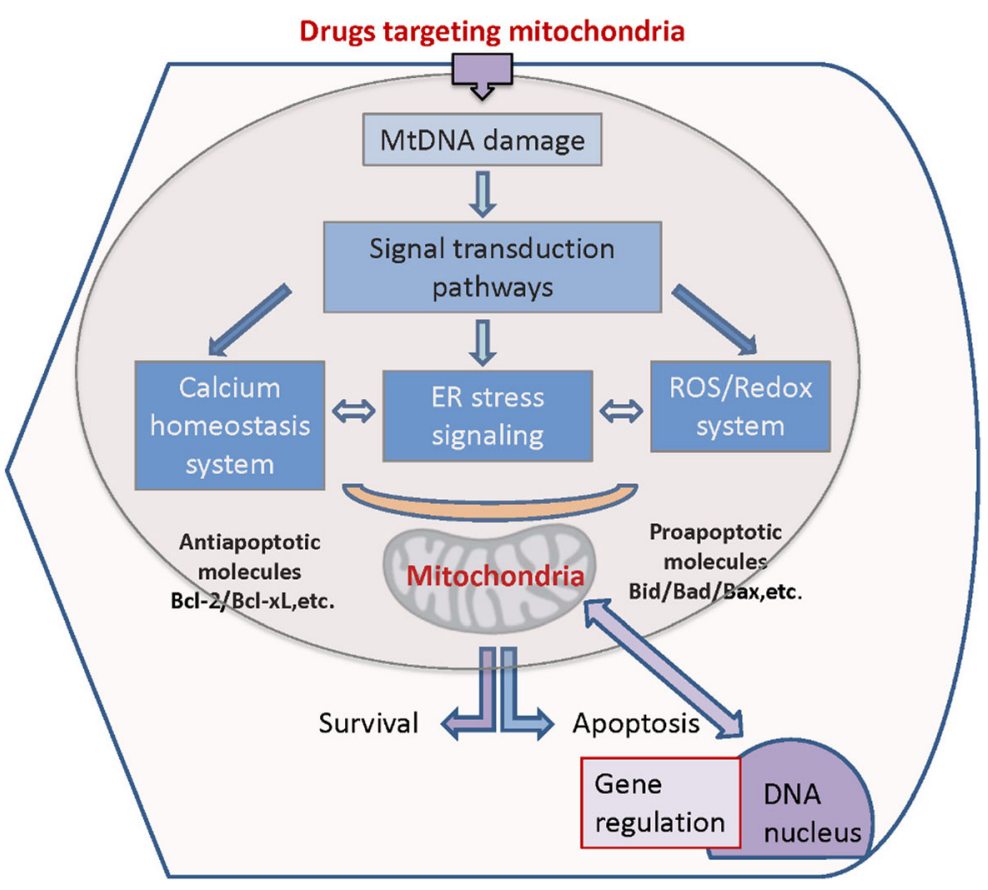

\section{Targeting mitochondria and the related diseases/ potential therapies}

MtDNA exists in numerous identical copies per cell (homoplasmy), but can also exist in multiple forms within the same tissue or cell (heteroplasmy). Given that each cell has many mitochondria, a significant amount of mitochondrial genome is located within each cell. Therefore, this is of paramount importance as far as drugs targeting mitochondria are concerned. Furthermore, we should try to understand whether drugs can directly or indirectly interact with MtDNA, leading to the occurrence of MtDNA mutations and dysfunction. Cell biology and toxicology should focus on the functional cross talk between signal networks and regulators in the upstream or downstream pathways of MtDNA. For example, the nuclear MYST family acetyl transferase MOF can regulate the expression of respiratory genes from both nuclear and mitochondrial DNA and the oxidative phosphorylation by binding with MtDNA (Chatterjee et al. 2016). MOF acts as a dualtranscriptional regulator of nuclear and mitochondrial genomes in the process of epigenetics and metabolism and binds with MtDNA under the activation of KAT8 regulatory NSL complex subunit 3. Intracellular and extracellular stimuli or drugs may influence the amount and activation of MOF, resulting in respiratory and
MtDNA transcriptional dysfunction and abnormal metabolism as well as diseases, e.g., hypertrophic cardiomyopathy and cardiac failure (Chatterjee et al. 2016).Mutations of MtDNA does not only lead to mitochondrial diseases, but is also present in other diseases, e.g., inflammation or cancer (Zhu and Wang 2017). Further evidence demonstrates that reduced MtDNA copy number altered mitochondrial aberrations, fertility phenotype, testes morphology, and spermatocyte proteome profiles of spermatocytes and spermatids in testes, which were prevented by increasing MtDNA copy number (Jiang et al. 2017). This indicates that the correction of MtDNA abnormality can be an alternative of potential therapies. Targeting mitochondria through the use of therapies used in treating patients with mitochondrial DNA diseases is informative of potential drug targets within the mitochondrion.

We can conclude that MtDNA plays an irreplaceable role in biological processes the mitochondria can regulate. Mitochondria-based key principles will continue be one of the themes that Cell Biology and Toxicology will discuss and investigate. It is expected to know how drugs can initiate mitochondrial dysfunction, the role of nuclear messages in regulating MtDNA, and how mitochondria communicate between or with other cells. Further studies are crucial to discover how mitochondria control the process of immune response, autophagy/ 
mitophagy, genome activation, and cell interaction. It is also needed to innovate how the transcription is started and terminated within mitochondria, the cytosolic proteins and other organelles interact with mitochondria, and MtDNA regulates the function of mitochondrial respiratory megacomplexes. We should aim to the mechanisms of how MtDNA dominates and maintains mitochondrial and cellular hemostasis in multiple conditions, activates inflammasome and inflammatory reactions, and contributes to the pathogenesis of diseases and carcinogenesis of malignances. Thus, we believe that Cell Biology and Toxicology will play a critical role in understanding molecular mechanisms of mitochondriaassociated biology, disease, and toxicology.

\section{References}

Bao L, Diao H, Dong N, Su X, Wang B, Mo Q, et al. Histone deacetylase inhibitor induces cell apoptosis and cycle arrest in lung cancer cells via mitochondrial injury and p53 upacetylation. Cell Biol Toxicol. 2016;32(6):469-82. https://doi.org/10.1007/s10565-016-9347-8

Chatterjee A, Seyfferth J, Lucci J, Gilsbach R, Preiss1 S, Böttinger $\mathrm{L}$, et al. MOF acetyl transferase regulates transcription and respiration in mitochondria. Cell. 2016;167(3):722-738.e23. https://doi.org/10.1016/j.cell.2016.09.052.

Ganta KK, Mandal A, Chaubey B. Depolarization of mitochondrial membrane potential is the initial event in nonnucleoside reverse transcriptase inhibitor efavirenz induced cytotoxicity. Cell Biol Toxicol. 2017;33(1):69-82. https://doi.org/10.1007/s10565-016-9362-9.

Gao D, Zhu B, Sun H, Wang X. Mitochondrial DNA methylation and related disease. Adv Exp Med Biol. 2017;1038:117-32. https://doi.org/10.1007/978-981-10-6674-0_9.

Gardner K, Hall PA, Chinnery PF, Payne BA. HIV treatment and associated mitochondrial pathology: review of 25 years of in vitro, animal, and human studies. Toxicol Pathol. 2014; https://doi.org/10.1177/0192623313503519.

Hillen HS, Parshin AV, Agaronyan K, Morozov YI, Graber JJ, Chernev A, et al. Mechanism of transcription antitermination in human mitochondria. Cell. 2017;171(5): 1082-1093.e13. https://doi.org/10.1016/j.cell.2017.09.035.

Hou J, Eldeeb M, Wang X. Beyond deubiquitylation: USP30mediated regulation of mitochondrial homeostasis. Adv Exp Med Biol. 2017;1038:133-48. https://doi.org/10.1007 /978-981-10-6674-0 10.

Jiang M, Kauppila TES, Motori E, Li X, Atanassov I, FolzDonahue K, et al. Increased total MtDNA copy number cures male infertility despite unaltered MtDNA mutation load. Cell Metab. 2017;26(2):429-436.e4. https://doi.org/10.1016/j. cmet.2017.07.003.

Kikuchi S, Ninomiya T, Kohno T, Kojima T, Tatsumi H. Cobalt inhibits motility of axonal mitochondria and induces axonal degeneration in cultured dorsal root ganglion cells of rat. Cell Biol Toxicol. 2017; https://doi.org/10.1007/s10565-0179402-0.

Li Q, Qi F, Meng X, Zhu C, Gao Y. Mst1 regulates colorectal cancer stress response via inhibiting Bnip3-related mitophagy by activation of $\mathrm{JNK} / \mathrm{p} 53$ pathway. Cell Biol Toxicol. 2017; https://doi.org/10.1007/s10565-017-9417-6.

Liu F, Sanin DE, Wang X. Mitochondrial DNA in lung cancer. Adv Exp Med Biol. 2017;1038:9-22. https://doi.org/10.1007 /978-981-10-6674-0_2.

Lv J, Bhatia M, Wang X. Roles of mitochondrial DNA in energy metabolism. Adv Exp Med Biol. 2017;1038:71-83. https://doi.org/10.1007/978-981-10-6674-0_6.

Medvedev R, Hildt E, Ploen D. Look who's talking-the crosstalk between oxidative stress and autophagy supports exosomaldependent release of HCV particles. Cell Biol Toxicol. 2017;33(3):211-31. https://doi.org/10.1007/s10565-0169376-3.

Qian M, Spada C, Wang X. Approach, application, and bioethics of MtDNA sequencing in cancer. Adv Exp Med Biol. 2017;1038:23-38. https://doi.org/10.1007/978-981-106674-0_3.

Singireesu SSNR, Misra S, Mondal SK, Yerramsetty S, Sahu N, K SB. Costunolide induces micronuclei formation, chromosomal aberrations, cytostasis, and mitochondrial-mediated apoptosis in Chinese hamster ovary cells. Cell Biol Toxicol. 2017; https://doi.org/10.1007/s10565-017-9411-z.

Song D, Cretoiu D, Wang X. Mitochondrial DNA in telocytes. Adv Exp Med Biol. 2017;1038:55-70. https://doi. org/10.1007/978-981-10-6674-0_5.

Sun H, Shi W, Wang X. How far can mitochondrial DNA drive the disease? Adv Exp Med Biol. 2017;1038:1-8. https://doi. org/10.1007/978-981-10-6674-0_1.

Wang W, Hou J, Zhu Z, Fang H. Is mitochondrial cell fragility a cell weakness? Adv Exp Med Biol. 2017a;1038:107-16. https://doi.org/10.1007/978-981-10-6674-0_8.

Wang L, Liebmen MN, Wang X. Roles of mitochondrial DNA signaling in immune responses. Adv Exp Med Biol. 2017b;1038:39-53. https://doi.org/10.1007/978-981-106674-0_4.

Wyatt AW, Annala M, Aggarwal R, Beja K, Feng F, Youngren J, et al. Concordance of circulating tumor DNA and matched metastatic tissue biopsy in prostate cancer. J Natl Cancer Inst. 2017; 109(12). https://doi.org/10.1093/jnci/djx118.

Xu MM, Pu Y, Han D, Shi Y, Cao X, Liang H, et al. Dendritic cells but not macrophages sense tumor mitochondrial DNA for cross-priming through signal regulatory protein $\alpha$ signaling. Immunity. 2017;47(2):363-373.e5. https://doi.org/10.1016/j. immuni.2017.07.016.

Zhang L, Reyes A, Wang X. The role of DNA repair in maintaining mitochondrial DNA stability. Adv Exp Med Biol. 2017;1038:85-105. https://doi.org/10.1007/978-981-106674-0_7.

Zhu Z, Wang X. Significance of mitochondria DNA mutations in diseases. Adv Exp Med Biol. 2017;1038:219-30. https://doi. org/10.1007/978-981-10-6674-0_15. 